\title{
COMPLEX SYMMETRIC OPERATORS, SKEW SYMMETRIC OPERATORS AND REFLEXIVITY
}

\begin{abstract}
SEN ZHU
Abstract. Let $\mathscr{H}$ be a complex separable infinite-dimensional Hilbert space and $C$ be a conjugation on $\mathscr{H}$. Let $\mathscr{C}$ and $\mathscr{S}$ denote respectively the set of $C$-symmetric operators and the set of $C$-skew-symmetric operators on $\mathscr{H}$. It is proved that $\mathscr{C}$ and $\mathscr{S}$ are Roberts orthogonal to each other, and some distance formulas from an operator to the sets $\mathscr{C}, \mathscr{S}$ are obtained. We exhibit the annihilating relation between $\mathscr{C}$ and $\mathscr{S}$ by describing their preannihilators. As applications, it is shown that $\mathscr{S}$ is hyperreflexive and not transitive.
\end{abstract}

Mathematics subject classification (2010): Primary 47B99, 47A15, Secondary 47L05.

Keywords and phrases: Complex symmetric operator, skew-symmetric operator, preannihilator, reflexivity, transitivity.

\section{REFERENCES}

[1] G. BIRKhofF, Orthogonality in linear metric spaces, Duke Math. J. 1 (1935), no. 2, 169-172.

[2] J. B. ConwaY, A course in operator theory, Graduate Studies in Mathematics, 21. American Mathematical Society, Providence, RI, 2000.

[3] S. R. Garcia, Approximate antilinear eigenvalue problems and related inequalities, Proc. Amer. Math. Soc. 136 (2008), no. 1, 171-179 (electronic).

[4] S. R. GARCIA AND D. E. POORE, On the norm closure problem for complex symmetric operators, Proc. Amer. Math. Soc. 141 (2013), no. 2, 549-549.

[5] S. R. GARCIA AND D. E. POORE, On the norm closure of the complex symmetric operators: compact operators and weighted shifts, J. Funct. Anal. 264 (2013), no. 3, 691-712.

[6] S. R. Garcia, E. Prodan, And M. Putinar, Mathematical and physical aspects of complex symmetric operators, J. Phys. A: Math. Gen. 47 (2014), no. 35, 353001.

[7] S. R. GARCIA AND M. PutinaR, Complex symmetric operators and applications, Trans. Amer. Math. Soc. 358 (2006), no. 3, 1285-1315 (electronic).

[8] S. R. Garcia And M. Putinar, Complex symmetric operators and applications, II, Trans. Amer. Math. Soc. 359 (2007), no. 8, 3913-3931 (electronic).

[9] S. R. GARCiA AND W. R. Wogen, Complex symmetric partial isometries, J. Funct. Anal. 257 (2009), no. 4, 1251-1260.

[10] S. R. GARCIA AND W. R. Wogen, Some new classes of complex symmetric operators, Trans. Amer. Math. Soc. 362 (2010), no. 11, 6065-6077.

[11] K. GuO, Y. Ji, AND S. ZHU, A $C^{*}$-algebra approach to complex symmetric operators, Trans. Amer. Math. Soc. 367 (2015), no. 10, 6903-6942.

[12] K. GUO AND S. ZHU, A canonical decomposition of complex symmetric operators, J. Operator Theory 72 (2014), no. 2, 529-547.

[13] R. A. Horn And C. R. Johnson, Matrix analysis, Corrected reprint of the 1985 original, Cambridge University Press, Cambridge, 1990.

[14] K. Kliś-Garlicka And M. PTAK, k-hyperreflexive subspaces, Houston J. Math. 32 (2006), no. 1, 299-313 (electronic).

[15] K. Kliś-Garlicka And M. PTAK, C -symmetric operators and reflexivity, Oper. Matrices 9 (2015), no. $1,225-232$. 
[16] C. G. Li And T. T. Zhou, Skew symmetry of a class of operators, Banach J. Math. Anal. 8 (2014), no. 1, 279-294.

[17] C. G. Li And S. ZHU, Skew symmetric normal operators, Proc. Amer. Math. Soc. 141 (2013), no. 8, 2755-2762.

[18] B. D. RoBerTs, On the geometry of abstract vector spaces, Tôhoku Math. J. 39 (1934), 42-59.

[19] S. M. ZAGORODNYUK, On a J-polar decomposition of a bounded operator and matrices of $J$ symmetric and J-skew-symmetric operators, Banach J. Math. Anal. 4 (2010), no. 2, 11-36.

[20] S. M. ZAGORODNYUK, On the complex symmetric and skew-symmetric operators with a simple spectrum, Symmetry, Integrability and Geometry: Methods and Applications 7 (2011), 1-9.

[21] S. ZHU, Approximate unitary equivalence to skew symmetric operators, Complex Anal. Oper. Theory 8 (2014), no. 7, 1565-1580.

[22] S. ZHU, Skew symmetric weighted shifts, Banach J. Math. Anal. 9 (2015), no. 1, 253-272.

[23] S. ZHU, Approximation of complex symmetric operators, Math. Ann. 364 (2016), no. 1-2, 373-399.

[24] S. ZHU AND C. G. LI, Complex symmetry of a dense class of operators, Integral Equations Operator Theory 73 (2012), no. 2, 255-272.

[25] S. ZHU, C. G. LI, AND Y. Q. Ji, The class of complex symmetric operators is not norm closed, Proc. Amer. Math. Soc. 140 (2012), no. 5, 1705-1708.

[26] S. Zhu And J. ZhaO, The Riesz decomposition theorem for skew symmetric operators, J. Korean Math. Soc. 52 (2015), no. 2, 403-416. 